\title{
DOES A QUANTUM PARTICLE KNOW THE TIME?
}

\author{
Lev Kapitanski And IgOR Rodnianski \\ Department of Mathematics \\ Kansas State University \\ Manhattan, Kansas 66506
}

Consider the Hamiltonian $H=-(1 / 4 \pi)\left(\partial^{2} / \partial x^{2}\right)$ on the circle $\mathbb{T}=\mathbb{R} / \mathbb{Z}$. The unitary exponent $\exp i t H$ is the solution operator for the time-dependent Schrödinger equation

$$
\frac{1}{i} \frac{\partial}{\partial t} E+\frac{1}{4 \pi} \frac{\partial^{2}}{\partial x^{2}} E=0
$$

i.e., $\exp i t H E^{0}(x)$ is the solution of (0.1a) with the initial condition $E^{0}$. The distributional kernel of $\exp i t H$ can be written as a series,

$$
\langle x|\exp i t H| y\rangle=\sum_{n \in \mathbb{Z}} \mathbf{e}\left(\frac{n^{2} t}{2}+n(x-y)\right)
$$

where we use the notation $\mathbf{e}(z)=e^{2 \pi i z}$. We replace $(x-y)$ by $x$ and look at the function

$$
E(t, x)=\sum_{n \in \mathbb{Z}} \mathbf{e}\left(\frac{n^{2} t}{2}+n x\right)
$$

We will be interested in the regularity of $E(t, x)$ in $x$ at different times $t \in[0,2)$.

It will be convenient to view the functions on $\mathbb{T}$ as periodic functions on $\mathbb{R}$ of period 1. Then (0.2) is the solution of (0.1a) corresponding to the initial condition - a comb,

$$
E(0, x)=E^{0}(x):=\sum_{n \in \mathbb{Z}} \delta(x-n) .
$$


The usual framework for describing the regularity of the solutions of the Schrödinger equation (0.1a) is the scale $\left\{H^{s}, s \in \mathbb{R}\right\}$ of $L^{2}$-Sobolev spaces. Recall that a distribution $f(x)=\sum_{m} f_{m} \mathbf{e}(m x)$ belongs to the space $H^{s}$ if $\sum_{m}\langle m\rangle^{2 s}\left|f_{m}\right|^{2}<\infty$, where $\langle m\rangle=$ $\left(1+m^{2}\right)^{1 / 2}$. The exponent exp it $H$ is a continuous operator in each of the Sobolev spaces $H^{s}$. The comb-function $(0.1 \mathrm{~b})$ lies in $H^{s}$, where $s$ is any number less than $-1 / 2$. For every $t>0$, the solution (0.2), if viewed through the telescope of Sobolev spaces, has the same regularity in $x$ variable, namely, $E(t, \cdot) \in \cup_{s<-1 / 2} H^{s}$.

The Sobolev spaces are not the only function spaces that can be applied to the analysis of the solutions of the Schrödinger equation. Of special interest for us here will be the Besov spaces. We show that the regularity of $E(t, \cdot)$, when measured in the appropriate Besov spaces, changes with $t$. The most drastic difference in regularity is between the cases when $t$ is rational and when $t$ is irrational. Within the set of irrational times, although there exists a generic regularity for generic $t$, there are different thin classes of irrationals which prescribe their particular regularity to the fundamental solution. These classes are singled out and characterized by the behavior of the continued fraction expansions of their members.

Note, that when $t$ is in the upper half-plane, then $E(t, x)$ defined in $(0.2)$ is (essentially) Jacobi's theta-function. The well-known transformation properties of thetafunctions allow to express $E(t, x)$, when $t$ is rational, as a linear combination of $\delta$ functions sitting in a (depending on $t$ ) finite number of points $x$ on the circle. This completely answers the question of regularity at rational times.

If $t$ is irrational, the situation is more complicated. Our choice of Besov spaces to measure the regularity of $E(t, \cdot)$, requires the estimates in $L^{\infty}$ of the exponential sums of the form

$$
\sum_{n \in \mathbb{Z}} \chi\left(2^{-j}|n|\right) \mathbf{e}\left(\frac{n^{2} t}{2}+n x\right)
$$

for large $j$. Here $\chi$ is a cut-off function, which is supported on the interval $[1 / 2,2]$, and which is either smooth, or equals 1 on this interval. The exponential sums (0.3) have been studied extensively, especially during the last 90 years. Of particular importance for us are the results of [Hardy \& Littlewood, 1914] with subsequent developments of [Mordell, 1926], and [Fiedler, Jurkat \& Körner, 1977], and [Bombieri, 1990]. 
We should mention that K. I. Oskolkov have obtained some nice results on the regularity of certain solutions of the Schrödinger equation, which exhibit different behavior for rational and irrational times, see [Oskolkov, 1992] and further references to his works therein. However, the questions he discusses and the function spaces he uses are different from the ones we concern ourselves in the present paper.

Also related to what we are doing, are the studies of the value distribution properites of theta-sums in [Jurkat \& van Horn, 1981, 1982], [Sarnak, 1981, 1982], [Marklof, 1996], and the studies of the geometric patterns generated by theta-sums in [Dekking \& Mendès France, 1981], [Deshouillers, 1985], [Berry \& Hannay, 1987], [Berry \& Goldberg, 1988], [Coutsias \& Kazarinoff, 1987].

Our interest in the regularity of $E(t, x)$ stemmed, initially, from our previous work on the regularity of the fundamental solution for the time-dependent Schrödinger equation in $\mathbb{R}^{n}$ with a growing at infinity potential. There, the model problem is the follwoing:

$$
\frac{1}{i} \frac{\partial}{\partial t} E-\Delta_{x} E+|x|^{\rho} E=0, \quad t \in \mathbb{R}^{1}, x \in \mathbb{R}^{d} ; \quad E(0, x, y)=\delta(x-y)
$$

$d \geq 1$, and $\rho$ is a positive constant. The regularity of $E(t, x, y)$ depends on the rate of growth of the potential. If $\rho<2$, then $E(t, x, y)$ is $C^{\infty}$-smooth in $(t, x, y), t \neq 0$; see [Yajima, 1996], [Kapitanski \& Rodnianski]. In the case $\rho=2$ the solution, $E(t, x, y)$, is given by Mehler's formula and shows that the singularities reappear at resonant times $t=m \pi, m \in \mathbb{Z}$, while for all other $t$ the fundamental solution is smooth. This picture survives the perturbations of $|x|^{2}$ by functions growing slower than quadratically, [Kapitanski, Rodnianski, Yajima, 1997].

When $\rho>2$ and $d>1$, nothing is known about the regularity of $E(t, x, y)$ (except, of course, for what the standard energy estimates give). However, it is likely that the fundamental solution is nowhere smooth. This conjecture is supported by a remarkable recent result of $\mathrm{K}$. Yajima, who showed that in the one-dimensional case $(d=1)$, if $\rho>2$, then $E(t, x, y)$ is not even in the local Bessel-Sobolev space $\mathcal{L}_{1, l o c}^{1 / \rho}\left(\mathbb{R}^{3}\right)$, see [Yajima, 1996].

The initial boundary value problem (IBVP) for the Schrödinger equation may be viewed as the extreme limit case of $(0.4)$ as $\rho \rightarrow \infty$. Yajima's technique, when applied to the one-dimensional IBVPs, gives the corresponding nonsmoothness results. In particular, it turns out that the distributional kernel $E(t, x, y)$ of the operator $\exp \{-i t \mathcal{H}\}$, where $\mathcal{H}=-(1 / 4 \pi) d^{2} / d x^{2}$ on the interval $[0,1 / 2]$ with Dirichlet boundary conditions, 
i.e., the function $E(t, x, y)=4 \sum_{n=1}^{\infty} e^{-\pi i t n^{2}} \sin 2 \pi n x \sin 2 \pi n y$ is nowhere locally integrable (in $\mathbb{R}^{3}$ ), see [Yajima], Remark 4 . Our results will add to this by revealing the fine changes in regularity of $E(t, \cdot, \cdot)$ at different times $t$.

ACKNOWLEDGMENTS. This work was partially supported by NSF grant DMS9623520. The authors thank Peter Sarnak and Jens Marklof for valuable discussions. The second author also thanks the Institute for Mathematics and Its Applications at the University of Minnesota, and the organizers of the workshop "Emerging Applications of Number Theory" at IMA, for their hospitality.

\section{Statements of Results}

We start with the definitions of the Besov spaces we need to state the results. For reference on Besov spaces see [Bergh \& Löfström], [Triebel].

Let $\chi$ be a $C_{0}^{\infty}$ function on $\mathbb{R}_{+}$with the following properties:

$$
\operatorname{supp} \chi=\left[2^{-1}, 2\right],
$$

and

$$
\sum_{j=-\infty}^{\infty} \chi\left(2^{-j} \xi\right)=1, \quad \forall \xi>0
$$

Define the functions

$$
\chi_{j}(\xi)=\chi\left(2^{-j} \xi\right), \quad j=1,2, \ldots ; \quad \chi_{0}(\xi)=1-\sum_{j=0}^{\infty} \chi_{j}(\xi) .
$$

With each of these functions we associate an operator $K_{j}$, which maps a distribution $f(x)=\sum_{m} f_{m} \mathbf{e}(m x)$ to a (finite) exponential sum,

$$
K_{j}(f)(x)=\sum_{m=-\infty}^{\infty} \chi_{j}(|m|) f_{m} \mathbf{e}(m x), \quad j=0,1, \ldots
$$

We define the Besov spaces $B_{u, v}^{s}$ on $\mathbb{T}$ for the following values of parameters $s, u$, and $v$ : $-\infty<s<+\infty, 1 \leq u, v \leq+\infty$. By definition, the space $B_{u, v}^{s}$ is composed of all distributions $f(x)=\sum_{m} f_{m} \mathbf{e}(m x)$ such that the following norm is finite:

$$
\begin{array}{ll}
\|f\|_{B_{u, v}^{s}} & =\left(\sum_{j=0}^{\infty} 2^{s j v}\left\|K_{j}(f)(\cdot)\right\|_{L^{u}}^{v}\right)^{\frac{1}{v}}, \quad \text { if } \quad v<\infty, \\
\|f\|_{B_{u, \infty}^{s}} & =\sup _{j \geq 0} 2^{s j}\left\|K_{j}(f)(\cdot)\right\|_{L^{u}},
\end{array}
$$


Along with the standard Besov spaces $B_{u, v}^{s}$, we use what we call the rough Besov spaces $\lceil B\rceil_{u, v}^{s}$. Their definition differs from that of $B_{u, v}^{s}$, practically, only in the choice of the cut-off function: instead of a smooth $\chi$, we take a step-function $\lceil\chi\rceil$ - the characteristic function of the interval $\left[2^{-1}, 2\right]$. To be more precise, define the operators $\lceil K\rceil_{j}$ for $j=0,1, \ldots$, as follows:

$$
\begin{array}{r}
\lceil K\rceil_{0}(f)(x)=\sum_{m=-2}^{2} f_{m} \mathbf{e}(m x) ; \\
\lceil K\rceil_{j}(f)(x)=\sum_{2^{j-1}<|m| \leq 2^{j+1}} f_{m} \mathbf{e}(m x), \quad j>0 .
\end{array}
$$

The norm in $\lceil B\rceil_{u, v}^{s}$ is defined as in (1.4), with $K_{j}$ replaced by $\lceil K\rceil_{j}$. It is known that if $1<u<\infty$, then the spaces $B_{u, v}^{s}$ and $\lceil B\rceil_{u, v}^{s}$ consist of the same distributions, and the norms are equivalent (see [Lizorkin, 1977], [Triebel, 2.5.4 and 9.1.3]). Recall, also, that $B_{2,2}^{s}=\lceil B\rceil_{2,2}^{s}=H^{s}(\mathbb{T})$.

In this paper we use the spaces $B_{\infty, \infty}^{s}$ and $\lceil B\rceil_{\infty, \infty}^{s}$ most of all. Therefore, we make a simpler notation for them: $B_{\infty}^{s}$ and $\lceil B\rceil_{\infty}^{s}$.

We shall say that a distribution $f$ belongs to $B_{\infty}^{s}$ sharp if $f \in B_{\infty}^{s}$ and $f \notin \cup_{\epsilon>0} B_{\infty}^{s+\epsilon}$. A similar convention will be in force for the spaces $\lceil B\rceil_{\infty}^{s}$ and $H^{s}$.

Closely related to the persistence of regularity of solutions of (0.1a) in $H^{s}$ is the following fact.

Remark 1.1. For all $t$, the distribution $E(t, \cdot)$ belongs to $B_{2, \infty}^{-1 / 2}$ sharp.

Remark 1.2. That $E(t, \cdot)$ belongs to the Besov space $\lceil B\rceil_{\infty}^{-\alpha}$ is equivalent to the existence of a constant $\lceil C\rceil$ such that

$$
\sup _{x}\left|\sum_{n=2^{j-1}}^{2^{j+1}} \mathbf{e}\left(\frac{n^{2} t}{2}+n x\right)\right| \leq\lceil C\rceil 2^{\alpha j}, \quad \text { for all sufficiently large } j \text {. }
$$

That $E(t, \cdot)$ belongs to the Besov space $B_{\infty}^{-\alpha}$ is equivalent to the existence of a constant $C$ such that

$$
\sup _{x}\left|\sum_{n=2^{j-1}}^{2^{j+1}} \chi\left(2^{-j} n\right) \mathbf{e}\left(\frac{n^{2} t}{2}+n x\right)+\chi\left(2^{-j} n\right) \mathbf{e}\left(\frac{n^{2} t}{2}-n x\right)\right| \leq C 2^{\alpha j}
$$
for all sufficiently large $j$. 
The regularity properties of $E(t, \cdot)$ in Besov spaces $B_{\infty}^{s}$ and $\lceil B\rceil_{\infty}^{s}$ depend on the continued fraction representation of $t$. We refer the reader to [Khinchin, 1964] and [Schmidt, 1980] for the basic theory of continued fractions. Consider first the case of a rational $t=\frac{p}{q} \in[0,2)$. In this case $t$ has a finite continued fraction expansion:

$$
t=\frac{p}{q}=\left[a_{0}, a_{1}, \ldots, a_{n}\right]=a_{0}+\frac{1}{a_{1}+\frac{1}{\ldots \frac{1}{\frac{1}{a_{n}}}}} .
$$

Note, that the expansion is not unique: we also have $\frac{p}{q}=\left[a_{0}, a_{1}, \ldots, a_{n-1}, a_{n}-1,1\right]$, if $a_{n} \neq 1$, and $\frac{p}{q}=\left[a_{0}, a_{1}, \ldots, a_{n-1}+1\right]$, if $a_{n}=1$.

If $t=\left[a_{0}, a_{1}, \ldots, a_{n}\right]$, then the numbers $p_{k} / q_{k}=\left[a_{0}, a_{1}, \ldots, a_{k}\right], k=1, \ldots, n-1$, are the corresponding convergents, and $p_{n}=p, q_{n}=q$.

Theorem I. Let $t \in[0,2)$ be a rational number, and $t=\frac{p}{q}$ - its simple fraction representation. Let $\frac{p_{k}}{q_{k}}, k=1, \ldots, n-1$, be partial convergents to $t$ determined by a finite continued fraction $\left[a_{0}, a_{1}, \ldots, a_{n}\right]$ with an odd number of quotients (i.e., $n$ is even). Then,

1) (formula)

$$
E\left(\frac{p}{q}, x\right)=\frac{\varkappa_{0}(t)}{\sqrt{q}} \mathbf{e}\left(-\frac{1}{2} q_{n-1} q x^{2}+\frac{1}{2} q \eta x-\frac{1}{8} \xi \eta\right) \sum_{n} \delta\left(\frac{n+\frac{1}{2} \xi}{q}-x\right)
$$

where

$$
\xi=p \cdot q(\bmod 2), \quad \eta=p_{n-1} \cdot q_{n-1}(\bmod 2),
$$

and $\varkappa_{0}(t)$ is an eighth root of 1 ;

2) (regularity)

$$
E(t, \cdot) \in B_{\infty}^{-1} \cap\lceil B\rceil_{\infty}^{-1} \quad \operatorname{sharp}
$$

When $t \in(0,2)$ is irrational, its continued fraction expansion $t=\left[a_{0}, a_{1}, \ldots, a_{n}, \ldots\right]$ is infinite and unique.

The regularity of $E(t, \cdot)$ for generic irrational $t$ is given by the following 


\section{Theorem II.}

(i) For almost all irrational $t, E(t, \cdot) \in \cap_{\varepsilon>0}\left(B_{\infty}^{-1 / 2-\varepsilon} \cap\lceil B\rceil_{\infty}^{-1 / 2-\varepsilon}\right)$.

(ii) If $t$ is an irrational number with bounded quotients, i.e., there is a constant $C>0$ such that $a_{n} \leq C$, for all $n$, then $E(t, \cdot) \in B_{\infty}^{-1 / 2} \cap\lceil B\rceil_{\infty}^{-1 / 2}$ sharp.

(iii) There is no $t$ for which $E(t, \cdot)$ belongs to $B_{\infty}^{(-1 / 2)+\varepsilon} \cup\lceil B\rceil_{\infty}^{(-1 / 2)+\varepsilon}$ with any positive $\varepsilon$.

We now define a few (narrower) classes of irrational numbers using restrictions on the growth of the denominators $q_{n}$ of their convergents $p_{n} / q_{n}$.

For $\sigma \geq 0$, denote by $\mathcal{I}(\leq \sigma)$ the set of all irrational $t$ such that for each of them there exists a constant $C_{t}$ such that

$$
q_{n+1} \leq C_{t} q_{n}^{1+\sigma}, \quad \text { for all sufficiently large } n
$$

Denote by $\mathcal{I}(\geq \sigma)$ the set of all irrational $t$ such that for each of them there exists a constant $c_{t}>0$ such that

$$
q_{n+1} \geq c_{t} q_{n}^{1+\sigma}, \quad \text { for an infinite number of } n \text {. }
$$

Finally, denote $\mathcal{I}(\sigma)=\mathcal{I}(\leq \sigma) \cap \mathcal{I}(\geq \sigma)$.

\section{Theorem III.}

(i) If $t \in \mathcal{I}(\leq \sigma)$, then

$$
E(t, \cdot) \in B_{\infty}^{-\frac{1+\sigma}{2+\sigma}} \cap\lceil B\rceil_{\infty}^{-\frac{1+\sigma}{2+\sigma}}
$$

(ii) If $t \in \mathcal{I}(\geq \sigma)$, then

$$
E(t, \cdot) \notin\left(\cup_{\epsilon>0} B_{\infty}^{-\frac{1+\sigma}{2+\sigma}+\epsilon}\right) \cup\left(\cup_{\epsilon>0}\lceil B\rceil_{\infty}^{-\frac{1+\sigma}{2+\sigma}+\epsilon}\right)
$$

(iii) If $t \in \mathcal{I}(\sigma)$, then

$$
E(t, \cdot) \in B_{\infty}^{-\frac{1+\sigma}{2+\sigma}} \cap\lceil B\rceil_{\infty}^{-\frac{1+\sigma}{2+\sigma}} \operatorname{sharp}
$$




\section{Estimates For Exponential Sums}

In this section we study two basic exponential sums,

$$
\lceil S\rceil_{M}^{N}(t, x)=\sum_{M \leq|n| \leq N} \mathbf{e}\left(\frac{n^{2} t}{2}+n x\right),
$$

and

$$
S_{M}^{N}(t, x)=\sum_{M \leq|n| \leq N} \omega_{n} \mathbf{e}\left(\frac{n^{2} t}{2}+n x\right) .
$$

The first sum is needed for the estimates of the norm of $E(t, \cdot)$ in the space $\lceil B\rceil_{\infty}^{s}$. The second is for the space $B_{\infty}^{s}$. There, the rôle of coefficients $\omega_{n}$ will be assigned to $\chi\left(2^{-j}|n|\right)$, see (0.3). However, in this section we do not restrict ourselves to this particular choice of $\omega_{n}$.

The estimates from above on $\left|\sum_{n=1}^{N} \mathbf{e}\left(\frac{n^{2} t}{2}+n x\right)\right|$ go back to [Hardy \& Littlewood]. They introduced the method of an approximate functional equation for incomplete thetasums. This method was developed further by [Fiedler, Jurkat \& Körner], who established, in particular, the result we need, Theorem 2.1 below. This result was later proved by [Bombieri] using a different technique (of maximal operators and Hunt-Carleson theorem).

Theorem 2.1. Let $t$ be real, and $\left|t-\frac{p}{q}\right| \leq \frac{1}{q^{2}}$, for some co-prime integers $p$ and $q$. Then there exists a constant $C>0$ such that, for all real $x$,

$$
\left|\sum_{n=1}^{N} \mathbf{e}\left(\frac{n^{2} t}{2}+n x\right)\right| \leq C\left(\frac{N}{\sqrt{q}}+\sqrt{q}\right)
$$

for any integer $N>0$.

This theorem implies immediately the following estimate.

Corollary 2.2. In the assumptions of Theorem 2.1,

$$
\left\|\lceil S\rceil_{M}^{N}(t, \cdot)\right\|_{L^{\infty}} \leq\lceil C\rceil\left(\frac{N-M}{\sqrt{q}}+\sqrt{q}\right),
$$

for all integers $M$ and $N>M$.

The estimate from above on the sum (2.2) is given by the following theorem (compare [Bourgain, Lemma 3.18]). 
Theorem 2.3. Let $t$ be real, and $\left|t-\frac{p}{q}\right| \leq \frac{1}{q^{2}}$, for some co-prime integers $p$ and $q$. Assume that the coefficients $\omega_{n}$ satisfy the following conditions:

$$
\omega_{n}=0, \quad \text { for } n<M \text { and } n>N
$$

and

$$
\sum_{M}^{N}\left|\omega_{n+1}-\omega_{n}\right| \leq \varkappa
$$

Then

$$
\left\|\sum_{M}^{N} \omega_{n} \mathbf{e}\left(\frac{n^{2} t}{2}+n \cdot\right)\right\|_{L^{\infty}} \leq \varkappa\lceil C\rceil\left(\frac{N-M}{\sqrt{q}}+\sqrt{q}\right)
$$

where $\lceil C\rceil$ is the same as in (2.4).

Proof. Using summation by parts, we obtain

$$
\begin{aligned}
& \left|\sum_{M}^{N} \omega_{n} \mathbf{e}\left(\frac{n^{2} t}{2}+n x\right)\right|=\mid \sum_{M}^{N}\left(\omega_{n+1}-\omega_{n}\right) \sum_{k=M}^{n} \text { e }\left(\frac{k^{2} t}{2}+k x\right) \mid \\
\leq & \varkappa \sup _{M \leq n \leq N}\left|\sum_{k=M}^{n} \mathbf{e}\left(\frac{n^{2} t}{2}+k x\right)\right|,
\end{aligned}
$$

and (2.6) follows from (2.4).

Corollary 2.4. Let $t$ be real, and $\left|t-\frac{p}{q}\right| \leq \frac{1}{q^{2}}$, for some co-prime integers $p$ and $q$. Assume that the coefficients $\omega_{n}$ satisfy the following conditions:

$$
\omega_{n}=0, \quad \text { for }|n|<M \text { and }|n|>N
$$

and

$$
\sum_{M \leq|n| \leq N}\left|\omega_{n+1}-\omega_{n}\right| \leq \varkappa
$$

Then

$$
\left\|S_{M}^{N}(t, \cdot)\right\|_{L^{\infty}} \leq 2 \varkappa\lceil C\rceil\left(\frac{N-M}{\sqrt{q}}+\sqrt{q}\right) .
$$

For the sharpness part in Theorems I - III we need to estimate the exponential sums from below. We do this in two steps. First, we estimate $\lceil S\rceil_{M}^{N}(t, x)$ and $S_{M}^{N}(t, x)$ for rational $t$, and then show that the (suprema of the) sums for an irrational $t$ are close to those with a sufficiently good rational approximation to $t$. This approach is quite standard, see [Montgomery, Chapter 3]. 
Theorem 2.5. Let $\lceil S\rceil_{M}^{N}(t, x)$ and $S_{M}^{N}(t, x)$ be as in (2.1), (2.2). Assume that $\omega_{n}$ satisfies (2.5a). Let $p$ and $q$ be co-prime positive integers.

For each of four cases below, there exists $x=\frac{h}{q} \in[0,1]$, with integer $h$, such that

$$
\begin{aligned}
\left|S_{M}^{N}\left(\frac{p}{q}, x\right)\right| & \geq \frac{\sum_{n=M}^{N} \omega_{n}+\omega_{-n}}{\sqrt{2} \sqrt{N-M}, \quad \text { if } \quad} \quad \text { q } \geq N-M+1 ; \\
\left|S_{M}^{N}\left(\frac{p}{q}, x\right)\right| & \geq \frac{\sum_{n=M}^{N} \omega_{n}+\omega_{-n}}{\sqrt{2 q}}, \quad \text { if } \quad q<N-M+1 ; \\
\left|\lceil S\rceil_{M}^{N}\left(\frac{p}{q}, x\right)\right| & \geq \sqrt{2} \sqrt{N-M}, \\
\left|\lceil S\rceil_{M}^{N}\left(\frac{p}{q}, x\right)\right| & \geq \frac{\sqrt{2}(N-M)}{\sqrt{q}}, \quad \text { if } \quad q \geq N-M+1 ;
\end{aligned}
$$

Proof. Because of the orthogonality relations among the additive characters $(\bmod 2 q)$, we have the following equalities:

$$
\sum_{h=1-(M-1) p}^{2 q-(M-1) p}\left|S_{M}^{N}\left(\frac{p}{q}, \frac{h}{q}\right)\right|^{2}=2 q \sum_{M}^{N}\left|\omega_{n}\right|^{2}+\left|\omega_{-n}\right|^{2}
$$

if $q \geq N-M+1$, and

$$
\sum_{h=1-(M-1) p}^{2 q-(M-1) p}\left|S_{M}^{N}\left(\frac{p}{q}, \frac{h}{q}\right)\right|^{2}=2 q \sum_{k=1}^{2 q}\left|\sum_{m=0}^{\left[\frac{N-M+1}{2 q}\right]} \omega_{2 m q+k+M-1}+\omega_{-2 m q-k-M+1}\right|^{2},
$$

if $q<N-M+1$. Since

$$
\left|\sum_{M}^{N} \omega_{n}+\omega_{-n}\right|^{2} \leq 2(N-M) \sum_{M}^{N}\left|\omega_{n}\right|^{2}+\left|\omega_{-n}\right|^{2}
$$

(2.10a) yields

$$
\sum_{h=1-(M-1) p}^{2 q-(M-1) p}\left|S_{M}^{N}\left(\frac{p}{q}, \frac{h}{q}\right)\right|^{2} \geq q \frac{\left|\sum_{M}^{N} \omega_{n}+\omega_{-n}\right|^{2}}{N-M}
$$


Hence,

$$
\left|S_{M}^{N}\left(\frac{p}{q}, \frac{h}{q}\right)\right|^{2} \geq \frac{\left|\sum_{M}^{N} \omega_{n}+\omega_{-n}\right|^{2}}{2(N-M)},
$$

for at least one $h$, and (2.8a) follows.

Similarly, the inequality

$$
\begin{aligned}
\left|\sum_{M}^{N} \omega_{n}+\omega_{-n}\right|^{2}=\left|\sum_{k=1}^{2 q} \sum_{m=0}^{\left[\frac{N-M+1}{2 q}\right]} \omega_{2 m q+k+M-1}+\omega_{-2 m q-k-M+1}\right|^{2} \leq \\
\qquad q \sum_{k=1}^{2 q}\left|\sum_{m=0}^{\left[\frac{N-M+1}{2 q}\right]} \omega_{2 m q+k+M-1}+\omega_{-2 m q-k-M+1}\right|^{2},
\end{aligned}
$$

and $(2.10 \mathrm{~b})$, show that for at least one integer $h$ we have $(2.8 \mathrm{~b})$ with $x=\frac{h}{q}$.

The estimates (2.9) follow from (2.8) if we choose $\omega_{n}$ to be 1 for $n \in[-N,-M] \cup$ $[M, N]$, and 0 otherwise.

Theorem 2.6. Assume that $t$ and $t_{1}$ are such that

$$
\left|t-t_{1}\right|<\frac{K}{N^{2}}
$$

with some constant $K>0$. Then

$$
\lceil c\rceil_{1} \max _{x}\left|\lceil S\rceil_{M}^{N}\left(t_{1}, x\right)\right| \leq \max _{x}\left|\lceil S\rceil_{M}^{N}(t, x)\right| \leq\lceil c\rceil_{2} \max _{x}\left|\lceil S\rceil_{M}^{N}\left(t_{1}, x\right)\right|,
$$

and

$$
c_{1} \max _{x}\left|S_{M}^{N}\left(t_{1}, x\right)\right| \leq \max _{x}\left|S_{M}^{N}(t, x)\right| \leq c_{2} \max _{x}\left|S_{M}^{N}\left(t_{1}, x\right)\right|,
$$

for some constants $c_{1}, c_{2},\lceil c\rceil_{1}$, and $\lceil c\rceil_{2}$, which depend only on $K$.

Proof. The proof follows the same line as the proof of Theorem 3.3 of [Montgomery]. First, we define an auxiliary, period 2 function $f(\theta)$ :

$$
f(\theta)= \begin{cases}\mathbf{e}\left(\frac{t-t_{1}}{2}(2 N \theta)^{2}\right), & \text { for } \quad 0 \leq \theta \leq \frac{1}{2}, \\ \mathbf{e}\left(\frac{t-t_{1}}{2} N^{2}\right) & \text { for } \quad \frac{1}{2} \leq \theta \leq 1,\end{cases}
$$


and $f(\theta)=f(-\theta)$, for $-1 \leq \theta \leq 0$. Taking into account (2.11), it is easy to check that the total variation of the first derivative of $f$ is bounded, $\operatorname{Var} f^{\prime} \leq \gamma_{1}$, and the Fourier coefficients of $f$ decay as $m^{-2},|\hat{f}(m)| \leq \gamma_{2} / m^{2}$. Note, that the constants $\gamma_{1}$ and $\gamma_{2}$ do not depend on $t, t_{1}$, and $N$.

Choose an $x$ so that $\left|S_{M}^{N}(t, x)\right|=\max _{y}\left|S_{M}^{N}(t, y)\right|$. Then we have

$$
\begin{aligned}
& \left|S_{M}^{N}(t, x)\right|=\left|\sum_{n=M}^{N} \omega_{n} \mathbf{e}\left(\frac{n^{2} t}{2}+n x\right)+\omega_{-n} \mathbf{e}\left(\frac{n^{2} t}{2}-n x\right)\right|= \\
& \left|\sum_{n=M}^{N} \omega_{n} f\left(\frac{n}{2 N}\right) \mathbf{e}\left(\frac{n^{2} t_{1}}{2}+n x\right)+\omega_{-n} f\left(\frac{n}{2 N}\right) \mathbf{e}\left(\frac{n^{2} t_{1}}{2}-n x\right)\right|= \\
& \mid \sum_{m} \hat{f}(m)\left(\sum_{n=M}^{N} \omega_{n} \mathbf{e}\left(\frac{n^{2} t_{1}}{2}+n\left(x+\frac{m n}{2 N}\right)\right)+\omega_{-n} \mathbf{e}\left(\frac{n^{2} t_{1}}{2}-n\left(x-\frac{m n}{2 N}\right)\right) \mid=\right. \\
& \text { ( note, that } \hat{f}(m)=\hat{f}(-m)) \\
& \mid \sum_{m} \hat{f}(m)\left(\sum_{n=M}^{N} \omega_{n} \mathbf{e}\left(\frac{n^{2} t_{1}}{2}+n\left(x+\frac{m n}{2 N}\right)\right)+\omega_{-n} \mathbf{e}\left(\frac{n^{2} t_{1}}{2}-n\left(x+\frac{m n}{2 N}\right)\right) \mid=\right. \\
& \left|\sum_{m} \hat{f}(m) S_{M}^{N}\left(t_{1}, \frac{x+m n}{2 N}\right)\right| \leq \max _{y}\left|S_{M}^{N}\left(t_{1}, y\right)\right| \cdot \sum_{m}|\hat{f}(m)| \leq c_{2} \max _{y}\left|S_{M}^{N}\left(t_{1}, y\right)\right| \text {. }
\end{aligned}
$$

The opposite inequality follows by reversing the rôles of $t$ and $t_{1}$. Also, (2.12a) is a particular case of $(2.12 \mathrm{~b})$.

\section{Proofs of Main Results}

\section{RATIONAL TIMES}

The fundamental solution $E(t, x)=\sum_{n} \mathbf{e}\left(\frac{n^{2} t}{2}+n x\right)$ is intimately related to the Jacobi theta function

$$
\vartheta(\tau, x, y)=\sum_{n} \mathbf{e}\left(\frac{\tau}{2}(n-y)^{2}+n x-\frac{1}{2} x y\right)
$$

Indeed, it is easy to see that

$$
\vartheta(\tau, x, y)=\mathbf{e}\left(-\frac{1}{2} y(x-\tau y)\right) E(\tau, x) .
$$


Recall, that by the well-known transformation property of $\vartheta$, [Eichler], for any unimodular matrix $g=\left(\begin{array}{ll}a & b \\ c & d\end{array}\right) \in S L(2, \mathbb{Z})$, we have

$$
\begin{aligned}
& \vartheta(\tau, x, y)= \\
& \varkappa(g)|c \tau+d|^{-\frac{1}{2}} \mathbf{e}\left(\frac{1}{4} \eta(a x+b y)-\xi(c x+d y)\right) \vartheta\left(\frac{a \tau+b}{c \tau+d}, a x+b y-\frac{1}{2} \xi, c x+d y-\frac{1}{2} \eta\right),
\end{aligned}
$$

where $\xi=a b \bmod 2, \eta=c d \bmod 2$, and $\varkappa(g)$ is an eighth root of $1 ; \varkappa(g)$ depends on the matrix $g$ and the choice of $\xi$ and $\eta$.

Let $t$ be a rational number in $(0,2), t=p / q, p$ and $q$ are co-prime. As it was explained in Section 1, we choose the finite continued fraction representation $p / q=$ $\left[a_{0}, a_{1}, \ldots, a_{n-1}, a_{n}\right]$ with even $n$. If $p_{n-1} / q_{n-1}=\left[a_{0}, a_{1}, \ldots, a_{n-1}\right]$, then $p q_{n-1}-$ $q p_{n-1}=1$, so, the matrix $g=\left(\begin{array}{cc}q & -p \\ q_{n-1} & -p_{n-1}\end{array}\right)$ is in $S L(2, \mathbb{Z})$. Using (3.3) with this $g$, and (3.2), we obtain

$$
\begin{gathered}
E(t, x)=\vartheta(t, x, 0)=\varkappa(g) \sqrt{q} \mathbf{e}\left(\frac{1}{4} x\left(q \eta-q_{n-1} \xi\right)\right) \vartheta\left(0, q x-\frac{1}{2} \xi, q_{n-1} x-\frac{1}{2} \eta\right)= \\
\varkappa(g) \sqrt{q} \mathbf{e}\left(\frac{1}{4}\left(q x-\frac{1}{2} \xi\right)\left(q_{n-1} x-\frac{1}{2} \eta\right)+\frac{1}{4} x\left(q \eta-q_{n-1} \xi\right)\right) E\left(0, q x-\frac{1}{2} \xi\right)= \\
\varkappa(g) \sqrt{q} \mathbf{e}\left(-\frac{1}{2} q q_{n-1} x^{2}+\frac{1}{2} q \eta x-\frac{1}{8} \xi \eta\right) \sum_{n} \delta\left(n-q x+\frac{1}{2} \xi\right) .
\end{gathered}
$$

This proves part 1) of Theorem I.

To prove part 2), we need an auxiliary result, which will be used in the proofs of other results as well.

Lemma 3.1. Let $\chi(\cdot)$ be a continuously differentiable, non-negative function on $\mathbb{R}_{+}$ with support in the interval $[1 / 2,2]$. Then, first,

$$
\sum_{n=0}^{\infty} \chi\left(2^{-j} n\right)=2^{j} \int_{0}^{\infty} \chi(y) d y+O(1), \quad \text { as } \quad j \rightarrow \infty
$$

and second,

$$
\sum_{n=2^{j-1}}^{2^{j+1}}\left|\chi\left(2^{-j}(n+1)\right)-\chi\left(2^{-j} n\right)\right| \leq \varkappa,
$$


for all $j \geq 0$, with some constant $\varkappa$.

Proof. The proof of (3.4) is by direct application of the well-known Euler-Maclaurin summation formula (see, e.g., [Edwards, Section 6.2]),

$$
\sum_{n=M}^{N} f(n)=\int_{M}^{N} f(y) d y+\frac{1}{2}(f(M)+f(N))+\int_{M}^{N}\left(y-[y]-\frac{1}{2}\right) f^{\prime}(y) d y,
$$

which is valid for any continuously differentiable function $f$ on the interval $[M, N]$.

To prove (3.5), apply the Newton-Leibniz formula.

Now, with the help of Lemma 3.1 and Remark 1.2, part 2) of Theorem I follows from Corollary 2.2, Corollary 2.4 and Theorem 2.5.

Let $t \in(0,2)$ be an irrational number and let $\left[a_{0}, a_{1}, a_{2}, a_{3}, \ldots\right]$ be its continued fraction expansion,

$$
t=a_{0}+\frac{1}{a_{1}+\frac{1}{a_{2}+\frac{1}{\ldots}}}
$$

The integers $a_{0}, a_{1}, a_{2}, \ldots$ can be found from the recurrent relations

$$
a_{k+1}=\left[\frac{1}{t_{k}}\right], \quad t_{k+1}=\frac{1}{t_{k}}-a_{k+1},
$$

and the initial conditions

$$
a_{0}=[t], \quad t_{0}=t-a_{0} .
$$

As usual, $[r]$ denotes the largest integer not exceeding $r$.

The finite parts $\left[a_{0}, a_{1}, a_{2}, \ldots, a_{n}\right], n=1,2, \ldots$, of our infinite continued fraction, sum up to the rational numbers $p_{n} / q_{n}$ - convergents of $t$. The numerators and denominators of the convergents can be found using the relations

$$
p_{k+1}=a_{k+1} p_{k}+p_{k-1}, \quad q_{k+1}=a_{k+1} q_{k}+q_{k-1},
$$

and the initial conditions

$$
p_{-1}=1, \quad q_{-1}=0, \quad p_{0}=a_{0}, \quad q_{0}=1
$$


Of course, $p_{n} / q_{n} \rightarrow t$. Also, we have

$$
\frac{1}{q_{n}\left(q_{n}+q_{n+1}\right)}<\left|t-\frac{p_{n}}{q_{n}}\right|<\frac{1}{q_{n} q_{n+1}}<\frac{1}{q_{n}^{2}} .
$$

For our purposes, it is important to know how fast the denominators $q_{n}$ grow. The celebrated result of Khinchin and Lévy (see [Khinchin, 1936], [Lévy, 1937]) answers this question in the following sense: for almost all $t$,

$$
\frac{\ln q_{n}}{n} \underset{n \rightarrow \infty}{\rightarrow} \ln \rho_{*}
$$

where $\rho_{*}$ is given by

$$
\ln \rho_{*}=\frac{\pi^{2}}{12 \ln 2}
$$

This means, in particular, that for any $t$ from the set defined by the Khinchin-Lévy theorem and for all sufficiently large $j$, there exists $\frac{p_{n}}{q_{n}}$, the $n^{t h}$ convergent to $t$, with $q_{n}=2^{j} \cdot 2^{j \epsilon_{j}}$, where $n=\left[j \log _{\rho_{*}} 2\right]$ and $\epsilon_{j} \rightarrow 0$ as $j \rightarrow \infty$. Hence, part (i) of Theorem II follows from Remark 1.2 and Corollaries 2.2 and 2.4 .

The equations (3.7) show that irrationals with bounded quotients belong to $\mathcal{I}(0)$. Thus, the second statement of Theorem II will follow from Theorem III. Since inequality (1.9) with $\sigma=0$ and $c_{t}=1$ holds true for every irrational $t$, as (3.7a) shows, the third statement of Theorem II follows from part (ii) of Theorem III. We, therefore, turn to the proof of Theorem III.

Proposition 3.2. If $t \in \mathcal{I}(\leq \sigma)$ for some $\sigma \geq 0$, then

$$
E(t, \cdot) \in B_{\infty}^{-\frac{1+\sigma}{2+\sigma}} \cap\lceil B\rceil_{\infty}^{-\frac{1+\sigma}{2+\sigma}}
$$

Proof. Recall, that $t \in \mathcal{I}(\leq \sigma)$ means that the denominators $q_{n}$ of the convergents to $t$ satisfy the inequality

$$
q_{n+1} \leq C_{t} q_{n}^{1+\sigma}
$$

for all sufficiently large $n$. In order to prove (3.10), we have to obtain the appropriate estimates (1.5) for truncated exponential sums. We achieve this by applying Corollaries 
2.2 and 2.4. The sums in (1.5a) and (1.5b) will be treated similarly, so we shall work only with the one that is involved in the definition of the space $\lceil B\rceil_{\infty}^{-\frac{1+\sigma}{2+\sigma}}$. Our goal is to show that there is a constant $\lceil C\rceil$ such that, for all $x$, the inequality

$$
\left|\sum_{m=2^{j-1}+1}^{2^{j+1}} \mathbf{e}\left(\frac{m^{2} t}{2}+m x\right)\right| \leq\lceil C\rceil 2^{\alpha j}
$$

holds true for all sufficiently large $j$, where

$$
\alpha=\frac{1+\sigma}{2+\sigma} .
$$

By Legendre's theorem, $\left|t-\left(p_{n} / q_{n}\right)\right| \leq 1 /\left(2 q_{n}^{2}\right)$, for any convergent $p_{n} / q_{n}$. Hence, Corollary 2.2 provides an estimate,

$$
\left|\sum_{m=2^{j-1}+1}^{2^{j+1}} \mathbf{e}\left(\frac{m^{2} t}{2}+m x\right)\right| \leq \tilde{C}\left(\frac{2^{j}}{\sqrt{q_{n}}}+\sqrt{q_{n}}\right)
$$

for an arbitrary $n$. It remains to show that

$$
\frac{2^{j}}{\sqrt{q_{n}}}+\sqrt{q_{n}} \leq C 2^{\alpha j}
$$

for every sufficiently large $j$, provided $n$ is chosen appropriately.

Define the exponents $s_{n}$ so that

$$
2^{s_{n}}=q_{n}
$$

In order that $(3.15 \mathrm{a})$ be true, the following inequalities ought to be satisfied: $\alpha \geq 1 / 2$ (which is the case when $\sigma \geq 0$ ), and

$$
\frac{s_{n}}{2 \alpha} \leq j \leq \frac{s_{n}}{2(1-\alpha)}
$$

We would like to have $(3.15 \mathrm{a})$ with $q_{n}$ replaced by $q_{n+1}$ as well. To achieve this, we impose a stronger requirement, which uses the assumption (3.11). Namely, we require that

$$
\frac{2^{j}}{\sqrt{q_{n+1}}}+\left(C_{t} q_{n}^{1+\sigma}\right)^{1 / 2} \leq C 2^{\alpha j}
$$


This inequality is satisfied provided

$$
\frac{s_{n}(1+\sigma)}{2 \alpha} \leq j \leq \frac{s_{n+1}}{2(1-\alpha)}
$$

Since $\alpha=\frac{1+\sigma}{2+\sigma}$, the right end of the interval (3.17a), $\frac{s_{n}}{2(1-\alpha)}$, coinsides with the left end of the interval $(3.17 \mathrm{~b}), \frac{s_{n}(1+\sigma)}{2 \alpha}$. Since the exponents $s_{n}$ grow to infinity, the above argument shows that the desired estimate (3.12) holds for all sufficiently large $j$. Proposition follows.

Proposition 3.3. If $t \in \mathcal{I}(\geq \sigma)$ for some $\sigma \geq 0$, then

$$
E(t, \cdot) \notin\left(\cup_{\epsilon>0} B_{\infty}^{-\frac{1+\sigma}{2+\sigma}+\epsilon}\right) \cup\left(\cup_{\epsilon>0}\lceil B\rceil_{\infty}^{-\frac{1+\sigma}{2+\sigma}+\epsilon}\right)
$$

Proof. Let $t \in \mathcal{I}(\geq \sigma)$. Recall, that this means that

$$
q_{n+1} \geq c_{t} q_{n}^{1+\sigma}
$$

for an infinite number of $n$ 's. We are going to prove that $E(t, \cdot) \notin \cup_{\varepsilon>0} B_{\infty}^{-\alpha+\epsilon}$, where $\alpha$ is defined in (3.13). It is sufficient to show that there exists an infinite number of $j$ 's, such that

$$
\sup _{x}\left|\sum_{m=2^{j-1}}^{2^{j+1}} \chi\left(2^{-j} m\right) \mathbf{e}\left(\frac{m^{2} t}{2}+m x\right)+\chi\left(2^{-j} m\right) \mathbf{e}\left(\frac{m^{2} t}{2}-m x\right)\right| \geq C 2^{\alpha j}
$$

We first note that if $p / q$ is such that

$$
\left|t-\frac{p}{q}\right| \leq K 2^{-2 j}
$$

then, by Theorem 2.6, we can replace $t$ by $p / q$ in (3.19). After this replacement, we can use Theorem 2.5 to get the following estimate:

$$
\begin{gathered}
\sup _{x}\left|\sum_{m=2^{j-1}}^{2^{j+1}} \chi\left(2^{-j} m\right) \mathbf{e}\left(\frac{m^{2} p}{2 q}+m x\right)+\chi\left(2^{-j} m\right) \mathbf{e}\left(\frac{m^{2} p}{2 q}-m x\right)\right| \\
\geq \tilde{c} \min \left\{2^{j / 2}, \frac{2^{j}}{\sqrt{q}}\right\}
\end{gathered}
$$


Note, that we have simplified the right sides of inequalities (2.8) by observing that

$$
\sum_{m=2^{j-1}}^{2^{j+1}} \omega_{m}+\omega_{-m}=2 \sum_{m=2^{j-1}}^{2^{j+1}} \chi\left(2^{-j} m\right) \geq \sqrt{2} \tilde{c} 2^{j}
$$

where the last inequality is a consequence of Lemma 3.1. Now, (3.19) will be proved if we show that

$$
\min \left\{2^{j / 2}, \frac{2^{j}}{\sqrt{q}}\right\} \geq C 2^{\alpha j}
$$

for an infinite number of $j$ 's, provided $p / q$ are chosen appropriately.

Our plan is to use the convergents $p_{n} / q_{n}$ in the above argument. We first check (3.20). Since $\left|t-\left(p_{n} / q_{n}\right)\right|<1 /\left(q_{n} q_{n+1}\right)$ for all $n$, we have

$$
\left|t-\frac{p_{n}}{q_{n}}\right|<\frac{1}{c_{t} q_{n}^{2+\sigma}}=\left(1 / c_{t}\right) 2^{-s_{n}(2+\sigma)},
$$

for those $n$, for which (3.18) takes place. Here and further on, $s_{n}=\log _{2} q_{n}$. Thus, to satisfy (3.20), it is sufficient to require that

$$
j \leq \frac{1}{2} s_{n}(2+\sigma) .
$$

Next, in order to have (3.22), it is sufficient to ask for the following inequality to hold:

$$
j \geq \frac{s_{n}}{2(1-\alpha)}-1=\frac{1}{2} s_{n}(2+\sigma) .
$$

The interval $\left[\frac{1}{2} s_{n}(2+\sigma)-1, \frac{1}{2} s_{n}(2+\sigma)\right]$ always contains an integer $j$, and, since $s_{n} \rightarrow \infty$ (we use only those $n$ for which (3.18) takes place), (3.19) is satisfied for an infinite number of $j$ 's. This completes the proof of the proposition.

\section{REFERENCES}

J. Bergh and J. Löfström, Interpolation spaces, an introduction, Springer, Berlin, 1976. M.V. Berry and J. Goldberg, Renormalization of curlicues, Nonlinearity 1 (1988), 1-26.

E. Bombieri, On Vinogradov's Mean Value Theorem and Weyl sums, Proceedings of the conference on "Automorphic forms and analytic number theory", Montréal, June 6-10, 1989, CRM, Montréal, 1990, pp. 7-24. 
J. Bourgain, Fourier transform restriction phenomena for certain lattice subsets and applications to nonlinear evolution equations.I. Schrödinger equations, Geom. Funct. Anal. 3 (1993), no. 2.

E. A. Coutsias and N. D. Kazarinoff, Disorder, renormalizability, theta functions and Cornu spirals, Physica D 26 (1987), 295-310.

F. M. Dekking and M. Mendès France, Uniform distribution modulo one: a geometric view point, J. Reine Angew. Math. 329 (1981), 143-153.

J.-M. Deshouillers, Geometric aspect of Weyl sums, Elementary and analytic theory of numbers, Banach Center publications, volume 17, PWN-Polish Scientific Publishers, Warsaw, 1985, pp. 75-82.

H. M. Edwards, Riemann's Zeta Function, Academic Press, New York and London, 1974.

M. Eichler, Introduction to the theory of algebraic numbers and functions, Academic Press, New York and London, 1966.

H. Fiedler, W. Jurkat and O. Körner, Asymptotic expansion of finite theta series, Acta Arithmetica 32 (1977), 129-146.

J. H. Hannay and M. V. Berry, Quantization of linear maps on a torus - Fresnel diffraction by a periodic grating, Physica D 1 (1980), 267-290.

G. H. Hardy and J. E. Littlewood, Some problems of Diophantine approximation, Acta Math 37 (1914), 155-239.

W. B. Jurkat and J. W. van Horn, The proof of the central limit theorem for theta sums, Duke Math. J. 48 (1981), 873-885.

W. B. Jurkat and J. W. van Horn, On the central limit theorem for theta series, Michigan Math. J. 29 (1982), 65-77.

L. Kapitanski and I. Rodnianski, Regulated smoothing for Schrödinger evolution, International Math. Research Notices 2 (1996), 41-54.

L. Kapitanski, I. Rodnianski and K. Yajima, On the fundamental solution of a perturbed harmonic oscillator, TMNA 9 (1997), no. 1, 77-106.

A. Ya. Khinchin, Zur metrischen Kettenbruchtheorie, Compositio Mathematica 3 (1936), no. 2, 275-285.

A. Ya. Khinchin, Continued fractions, Translated from the 3rd Russian edition of 1961, The University of Chicago Press, 1964.

P. Lévy, Théorie de l'addition des variables aléatoires, Paris, 1937.

P. I. Lizorkin, On bases and multipliers for the spaces $B_{p, \Theta}^{r}(\Pi)$, Trudy Mat. Inst. Steklov 
143 (1977), 88-104.

J. Marklof, Limit theorems for theta sums, see this volume; Limit theorems for theta sums with applications in quantum mechanics, Dissertation, Ulm, 1997.

H. L. Montgomery, Ten lectures on the interface between analytic number theory and harmonic analysis, CBMS, Regional Conference Series in Mathematics, Number 84, American Mathematical Society, Providence, Rhode Island, 1994.

L.J. Mordell, The approximate functional formula for the theta function, J. London Math. Society 1 (1926), 68-72.

K. I. Oskolkov, A class of I. M. Vinogradov's series and its applications in harmonic analysis, Springer Series in Computational Mathematics, 19, Progress in Approximation Theory, an International perspective (A. A. Gonchar and E. B. Staff, eds.), Springer-Verlag, New York, 1992.

P. Sarnak, Asymptotic behavior of periodic orbits of the horocycle flow and Eisenstein series, Comm. Pure Appl. Math. 34 (1981), 719-739.

P. Sarnak, Class numbers of indefinite binary quadratic forms, J. Number Theory $\mathbf{1 5}$ (1982), 229-247.

Schmidt, Diophantine approximation, Lecture Notes in Mathematics, 785, SpringerVerlag, Berlin Heidelberg New York, 1980.

H. Triebel, Theory of function spaces, Birkhäuser, Basel, 1983.

K. Yajima, Smoothness and non-smoothness of the fundamental solution of time dependent Schrödinger equations, Comm. Math. Phys. 181 (1996), no. 3, 605-629. 